\title{
A Comparison of Hashing Schemes for Address Lookup i n Computer Networks
}

\author{
Raj Jain, Seni or Member
}

\begin{abstract}
Using a trace of address references, we com- hashing al lows us to chop up a big table into several pared the efficiency of several different hashing func-small subtables so that we can quickly find the inf ortions, such as cyclic redundancy checking (CRO) poly- mation once we have determed the subt able to search nom als, Fletcher checksum fol ding of address octetsfor. This determination is made using a mathematical us ing the exclusive-or operation, and bit extraction frofnunction, which maps the given key to hash cell $i$, as the address. Guidelines are provided for determi ni ngshown in Fi gure 1. The cell $i$ could then poi nt us to the the size of hash mask required to achieve a specified subtable of size $e_{i}$ G ven a trace of $R$ franes with $N$ level of perf or mance. distinct addresses and a hashtable of $M$ cells, the goal is to mi ni me the average number of lookups requi red per frane.
\end{abstract}

\section{INTRODUCTI ON}

If we performa regul ar bi nary search through al $1 \mathrm{Nad}-$ dresses, we need to perform $1+\log _{2}\left(\mathrm{~g} N\right.$ or $\log _{2}(2 N$

The trend toward networks becoming $\mathrm{l}$ arger and faster, lookups per frame. G ven an address that hashes to addresses becoming $l$ arger, has i mpelled a need to ex- $i$ th cell, we have to search through a subt able ofem $\mathrm{pl}$ ore alternatives for $\mathrm{f}$ ast address recognition. Thi Bies, which requires onl $\mathrm{y}_{2}\left(\mathrm{gg}_{i}\right)$ lookups. The total problemis actually a special case of the general problemunber of lookups saved is:

of searching through a $l$ ar ge dat a base and finding the inf or mation as soci at ed wi th a gi ven key. For exampl e, Datal i nk adapters on local area networks (LAN) need to

$$
\text { Number of lookups saved }=\sum_{i} r_{i}\left[\log \left(2 N-\log _{2}\left(2 n_{i}\right)\right]\right.
$$
recognize the milticast destination addresses of frames on the LAN Bridges, used to interconnect t wo or more LANE, have to recognize the destination addresses of every frane and decide quickly whether to receive the frame for forwarding. Routers in wi de area networks have to look through a large for warding database to decide the output link for a given destination address. Nane servers have the ulti mate responsibility for associ ating nanes to characteristics. In these appl ications, hashing al gorithmcan be used to search through a very large inf or mation base in constant time. We al so investigated caching as a possible sol ution but found that caching coul d be har nf ul insome cas es in the sense that the perf or mance woul $d$ be better wi thout caching [4].

To compare various hashing strategi es, we used a trace of destination addresses observed on an Ether net LAN in use. The trace consisted of $2.046 \mathrm{~m} l \mathrm{li}$ ion $\mathrm{franes}$ observed over a period of 1.09 hours. At ot al of $495 \mathrm{dis}-$ ti nct station addresses were observed, of whi ch 296 wer e seen in the destination fiel $d$.

Where, $r_{i}$ is the number of frames that has h to the $i \mathrm{th}$ cell $\sum r_{i}=R$ The net saving per frane is:

$$
\begin{aligned}
\text { Lookups saved per frane } & =\sum_{i}-\frac{r_{i}}{R} \log _{2}\left(\frac{n_{i}}{N}\right) \\
& =\sum_{i}-q_{i} \log _{2}\left(p_{i}\right)
\end{aligned}
$$

Here, $q=r_{i} / R$ denotes the $\mathrm{fracti}$ on of $\mathrm{franes}$ that hash to $i$ th cell, and $_{i} p=n_{i} / N$ is the fraction of addresses that hash to $i$ th cell. The goal of a hashing function is to maxi m ze the quantity $\sum-q_{i} \log _{2}\left(p_{i}\right)$. Notice that ${ }_{i} p$ and $q_{i}$ ar e not rel ated. In the special case of all addresses bei ng equal ly likely to be $\mathrm{r}$ ef er enceds qqual t $o_{i}$ pand the expression $\sum-p_{i} \log _{2}\left(p_{i}\right)$ woul $d$ be called the entropy of the hashing function. It is because of this sim il arity that we will call the quantity $q_{i} \log _{2}\left(p_{i}\right)$ the entropy or information content of the hashing $f$ unction. It is measured in units of 'bits.'

\section{Ha s hi ng Us i ng Addres s Bi t s}

\section{Hashing: Con cepts}

Whster's dictionary defines the word 'hash' as a verb "to chop (as meat and potatoes) into small pieces". Strange as it may sound, this is correct. Basically?

The si mpl est has hi ng nethod is to use a cert ai n number of bits, say $m$, fromt he address. For example, we could hash using bits $i, i+1, \ldots, i+m-1$ of the address to $2^{2}$ subtables. The nunber of lookups saved, as ne saw in the last section, is equal to the inf or mation entropy 
of the bits. For our trace, this is plotted in Figure 4 Ha $\mathbf{s} \mathbf{i} \mathbf{n}$ Us i ng CRC E ght curves corresponding to monsecutive bits with $m=1,2, \ldots, 8$ are pl otted. FromFi gure 2 , we observe that:

Another hashing function, already used in a few adapt ers, is to use bits fromt he cycl ic redundancy check (CRC) of the address. Using the 32-bit CRC pol yno-

1. Al 18 - bi t sequences between bi ts 0 and 24 have less mi al used on IEEE 802 LANs [2], we computed the inthan two bits of inf or mation.

f or mation content of mbit sequences consisting of bits

2. The bits $32 \mathrm{through} 39$, in general, have a hi gh $i$ through $i+m-1$ of the CRCf or $m=1,2, \ldots, 8$, and inf or mati on content.

$i=0,1, \ldots, 31$. Here, $i=0$ represent $\mathrm{s}$ the most $\mathrm{signif-}$

i cant bit of the CRC The results are shown in Fi gure

The first observation is not surprizing considering that 3 .

the first three octets of the IEEE 802 addresses used on IEEE 802 LANs are assigned by the IEEE and, thus, most stations have the same first three octets. The first two bits corresponding to the global/local assignment and mul ticast/uni cast addresses my be different in different addresses. G ven these two bits, ot her bits can be easily predicted. In mul tivendor envir onments the first 3 octets may have a little more information. However, in general, these bits are not good for hashing pur poses.

The second observation says that the fif th octet of the address has the hi ghest i nf or mati on in our envi ronment. This observation, if applicable, leads to the following types of conclusions:

1. Use the fifth octet as the hashing function. The bits in this octet would provide a maxi mumsavings in the number of lookups.

2. When comparing two addresses, compare the fifth octet first. If the addresses are not equal, the very first comparis on will fail more of ten than when

It is interesting to compare Fi gures 2 and 3 . Notice the f ol l owi ng:

1. Al most all 32 bits have a hi gh i nf or mat $\mathrm{i}$ on cont ent very close to one bit. Thus, it does not matter whi ch bit of the CRCwe use.

2. Al l curves are (al most) horizontal straight lines. Thus, the inf or mation content of all bit conbinations is identical. It does not matter which mbits are chosen for $m=1,2, \ldots$

3. The inf or mation content of mbits is approximately $m$ This means that CRC provides an al most opti mal has hing function. using ot her octets.

3. Use the ffth octet as the branching function at $\mathbf{5}$ the root of a tree database. If the addresses are stored in a tree or trie structure [5] and the address bits are used to decide the branch to be taken, This checksumis used in the ISO/OSI transport since using bits fromt his oct et woul d provi de maxi mum discrimination.

Wepeated the analysis with several ot her 8 -bit and 16- bit CRC polynom als. In general, we found that if a pol ynomial provides a good CRC, it can serve as an excellent hash function.

\section{Ha s hi ng Us i ng Fle t c her Che c ks}

4. Lbe the fifth octet as the load bal ancing function it is so easy to compute in sof tware. G ven an $n$ octet mess age $b[1] \ldots \mathrm{b}[\mathrm{n}]$, a two- octet checksum $C[0]$ and $\phi 1]$ is computed as fol lows:

for different paths. G ven several al ternati ve pat h\& [0] $=0 ; \mathbf{C}[1]=0$;

to a set of destinations, one way to bal ance the For $i=1$ step 1 until $n$ do

load on different paths is to decide the path based $\mathbf{c}[0]=\mathbf{c}[0]+\mathrm{b}[\mathrm{i}]$;

on a f ew bits of the address. This eli mi nates out- $\mathbf{C}[1]=\mathbf{C}[1]+\mathbf{c}[0]$;

of-order arrivals since all frames going to a parEndFor;

ticul ar destination fol low a single path and load bal ancing is achi eved by different dest i nat i ons us ing di fferent paths.

It should be obvious that the fif th octet may not be the The $i$ nf ormation in mconsecutive bits of address checks uns is shown in Fi gure 4. Thi s al so is a good hashing function. most inf or mative octet for all environments. Nonetheless, the above recommendations are usef ul providing that one uses the appropriate, most inf ormative octet inst ead. 
6 Ha s hi ng Us i ng Anot her Che ck snuFngure 7. In the figure, the number of addresses $k$ that a station may want to receive is pl otted along the

Another popul ar checksum al gorithm us ed to guard agai ns t ne mory errors in network address databases is [2]:

$$
\begin{gathered}
C=\quad M d \quad\left(2^{8}(4 b[1]+2 b[3]+b[5])+\right. \\
(4 b[2]+2 b[4]+b[6]), \mathfrak{B}-1)
\end{gathered}
$$

Here, $b[i]$ is the $i$ th octet of the address and $C$ is the 16- bit checks um Si nce we are not aware of its nane, we will call it the 'nod-checksum' The inf ormation content of the bits of this checksumare shown in Fi gure 5 . Notice that the mod-checksumis not as good a hashing function as the Fletcher checks umeven though it is nore compl ex to compute.

\section{Has hi ng Us i ng XOR Fol di ng}

The final alternative that we investigated is that of the strai ghtf orward excl usi ve- or operation on the six octets of the address to produce 8 bits.

$$
C=b[1] \oplus b[2] \oplus b[3] \oplus b[4] \oplus b[5] \oplus b[6]
$$

horizontal axis, and the probability of rejecting an unwanted $\mathrm{frame}$ is plotted al ong the vertical axis. Eight curves corresponding to mask size $M=2,4,8, \ldots$, 128, 512 bits are shown. Fromfigure 7 , we observe the f ol l owing:

1. There is some fil tering even with small masks. For example, if one wants to receive 10 addresses, an 8bit mask is expected to rej ect $26 \%$ of the unwant ed franes wi thout $f$ urther searching. Al though this rate is low, the point is that it is non-zero even though the maskize is less than the number of address es.

2. In general, it is better to have as $l$ arge a mask as possible. For example, if one wants to receive 10 addresses with a 512 - bit mask, $98 \%$ of the unwanted frames will be rejected wi thout further searching.

3. If the mask size is $l$ ar ge compared to the number of addresses to receive, that is, if $M \gg k$, the curves are li near and the unwanted-rejection rate is approxi mately $1-k / M$

The inf or mation content of the bits in the 'XOR fold' so obtai ned is shown in Figure 6 . To our surprise, this The last observation is hel pf ul in deciding the masksize. f unction, which is so simple to i mplement, is an excell ent Thus, if one wants to rej ect $80 \%$ of the unwantedframes, hashing function. the maskize shoul d be 5 ti nes the number of addresses desired.

\section{Mas k Si ze for an Address Fi l t e r}

In this section, we bri efly address the probl emof finding the size of the hash mask required to get a desired level showed that the number of lookups saved using of performance. Wassume that the filter consists of a hashing is equal to the information content of the bits si mple $M \times 1$ bit mask, that is, each hash table cell is of the hashed val ue and compared sever al hashing $\mathrm{f}$ unconly one bit wide. A hash function is used to mpthe tions. First, simple bit extractionfromthe address itself address to an index val ue $i$ in the range 0 through $M A 1$, provides a hashing function that is easy to implenent and if the $i$ th bit in the mask is set, the frame is accepted $\mathrm{n}$ hardware as well as sof tware. Second, bits extracted for further processing; otherwise, the frane is rejectefromthe CRCof the address can be used as a hashing Thi s is howhashing is used in several comercial nedi a functi on that is easy to i mplenent in hardware. Third, access controller (MAC) chi ps. Such a hash filter is a bits extracted fromthe Fletcher checksumcan be used perfect rejection fil ter in the sense that if the maskit tass a hashing function that is easy to implement in sof $t$ zero, we can be certain that the address is not wanted, ware. Finally, excl usive- or folding of the address octets and there is no need to search the address table.

The perf or mance of the fil ter is meas ured by the probability of an unwanted address being rejected by the fil - concl uded that CRCpol ynom al s are excell ent hashter. Wall this probability the unwanted-rejection ing functions. Fletcher's checksumand folding are also rate. If we assume that all addresses are equally likelyood hashing functions. The nod-checks um which is to be seen and that all mask cells are equally likely tomre complex to compute than Fletcher's checksum is be referred, then using the procedure described in [3], not as good as the latter. Although bit extraction is not we can compute the unwanted-rejection rate as show as good as other alternatives, it is the simplest. The 
choice between bit extraction and other alternatives is basically that of computing vs storage. If we can use excess memory, bit extraction with a fewmo bits may provi de the s ame inf or mat $i$ on as the checksumor fol ding wi th a fewless bits.

Wointed out that for a station wanting to receive $k$ addresses, the probability of rejecting unwanted frames us ing a simple $M \times 1$ bit mask is $1-k / M$ This al lows us to deci de the mask size required for a desired level of perf or mance.

\section{Ref e rences}

[1] J. G Fletcher, "An Arithmetic Checksumf or Serial Tr ans mi s si ons," I EEE Tr ans. on Communi cat i ons, Vol. COM30, No. 1, Januar y 1982, pp. 247- 252.

[2] The Ethernet - A Local Area Network: Data Link Layer and Physical Layer Specifications, Publ is hed j oi ntly by $\mathrm{D}$ gital, Intel, and Xer ox Cor p, Version 2. 0, Nve nber 1982, pp. 95- 96.

[3] R Jai n, "A Comparis on of Has hing Schenes f or Address Lookup in Computer Networks," DEC Technical Report, DEC TR 593, February, 1989, $17 \mathrm{pp}$.

[4] R Jain, "Characteristics of Destination Address Local ity in Computer Networks: A Compari s on of Caching Schemes," Computer Networks and ISDN Systens, Vol. 18 (1989/90), pp. 243- 254 .

[5] R Sedgewick, A gorithms, Addis on- Wsley, Reading, MA 1988.

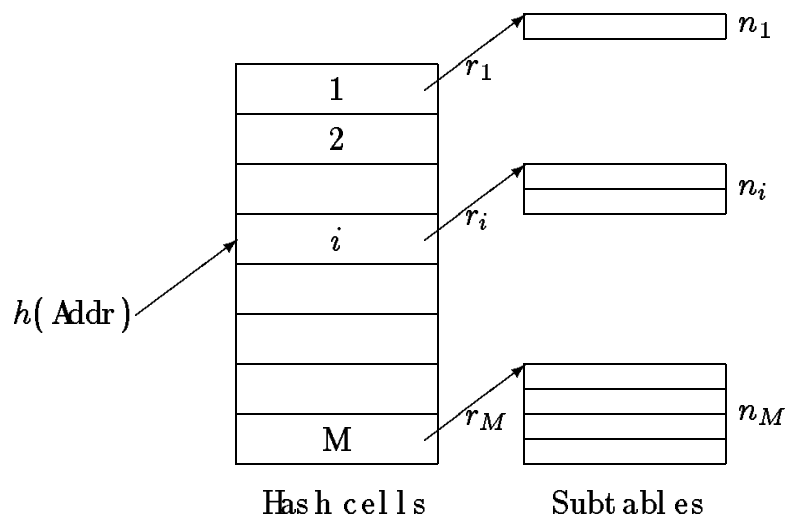

Fi gure 1: Has hi ng concepts.

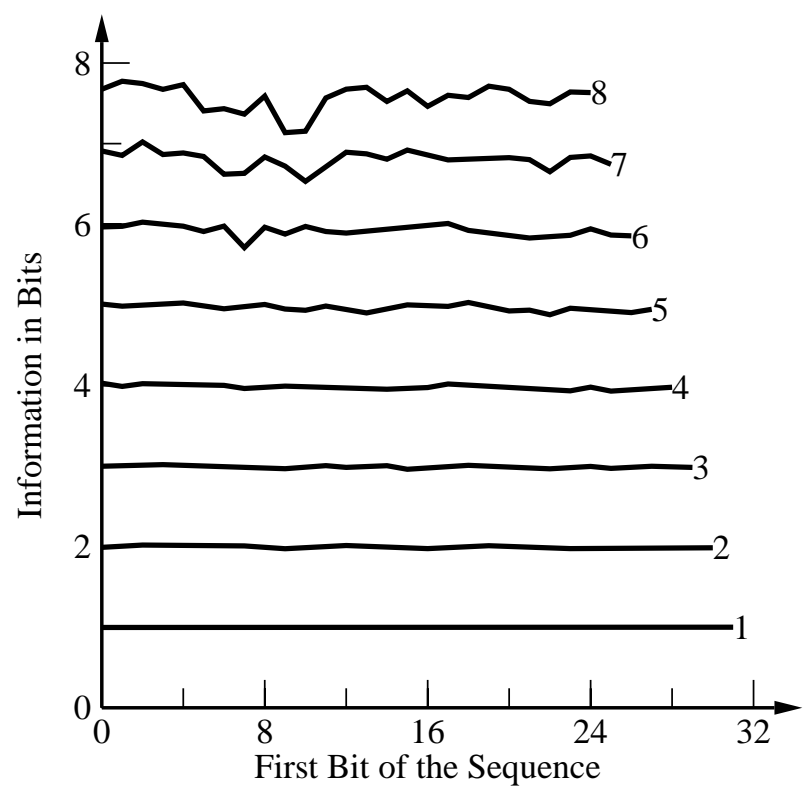

Fi gure 3: Inf or mati on in CRC bits . 

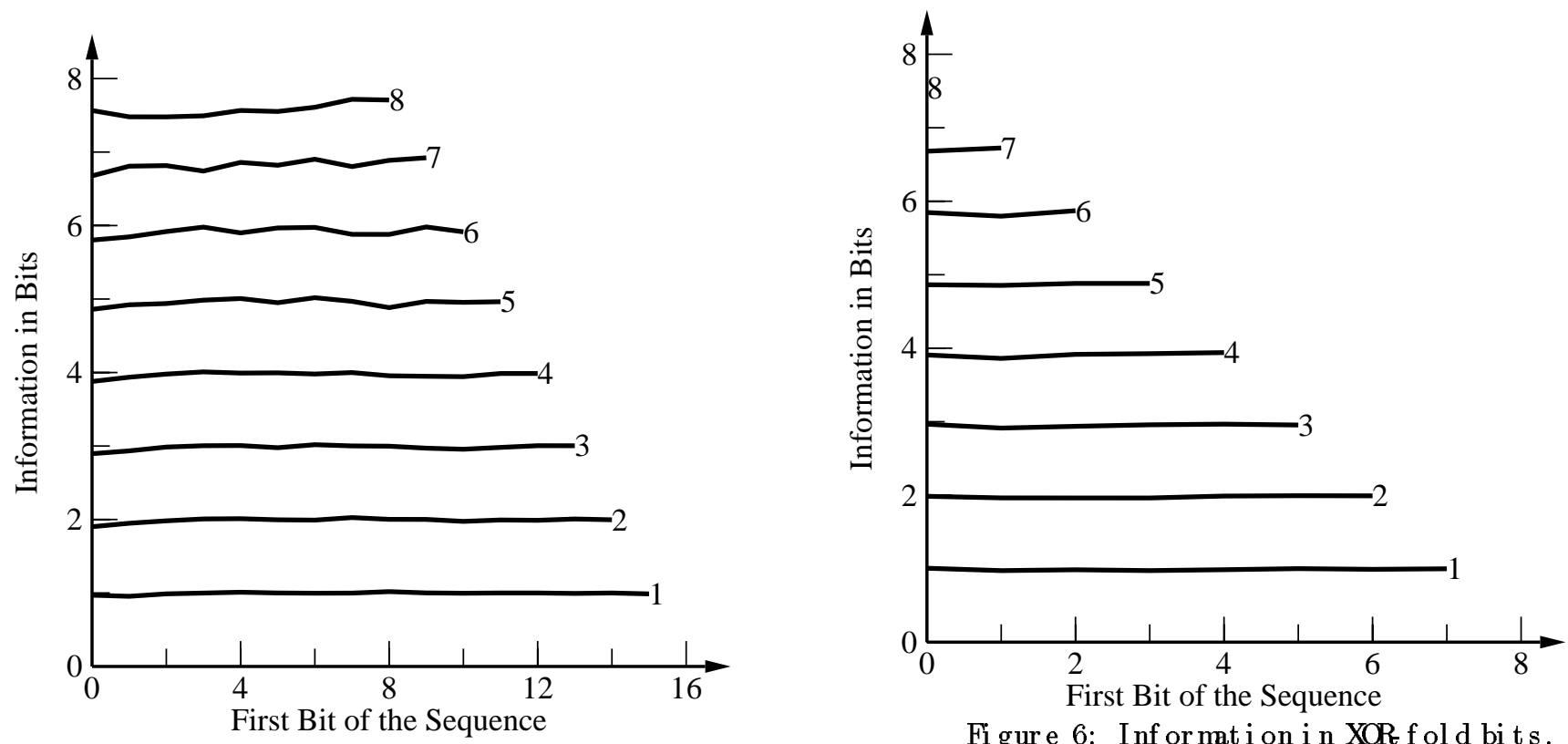

Fi gure 4: Inf or mation in Fl et cher checks umbits.
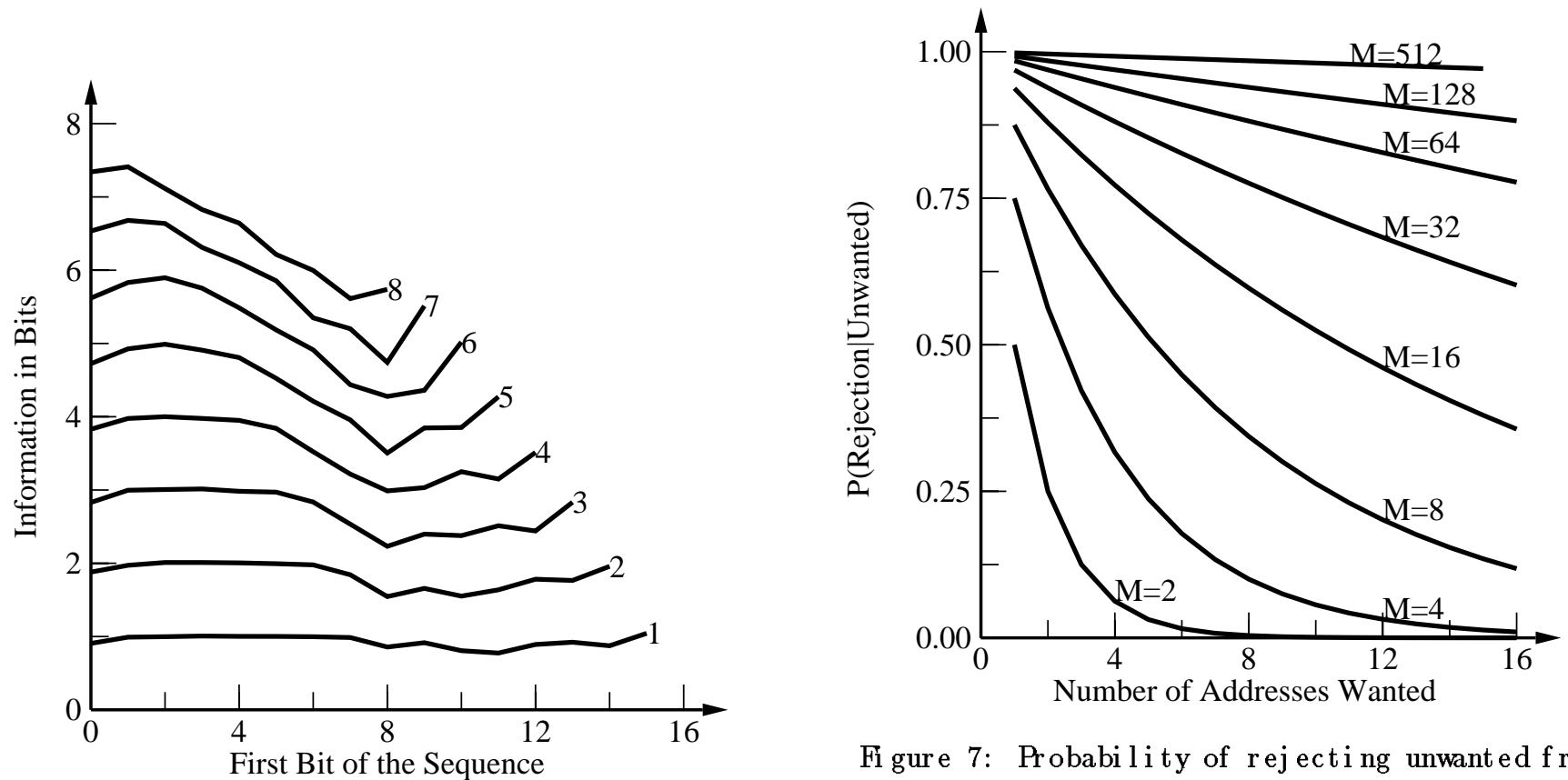

Fi gure 5: Inf or mati on in mod- checksumbits.

Figure 7: Probability of rejecting unwanted franes as a f unction of number of address wanted and the mask size $M$ 